\title{
ON EXTENSIONS OF GROUPS OF FINITE EXPONENT
}

\author{
PAVEL SHUMYATSKY \\ Department of Mathematics, University of Brasilia, Brasilia-DF, 70910-900 Brazil \\ e-mail:pavel@ipe.mat.unb.br
}

(Received 26 August, 2002; accepted 11 December, 2002)

\begin{abstract}
A well-known theorem of P. Hall says that if a group $G$ contains a normal nilpotent subgroup $N$ such that $G / N^{\prime}$ is nilpotent then $G$ is nilpotent. We give a similar sufficient condition for a group $G$ to be an extension of a group of finite exponent by a nilpotent group.
\end{abstract}

2000 Mathematics Subject Classification. 20F12.

1. Introduction. The following famous theorem is due to P. Hall [2].

THEOREM A. Let $N$ be a normal subgroup of a group $G$. If both $G / N^{\prime}$ and $N$ are nilpotent, then so is $G$. Futhermore, the nilpotency class of $G$ does not exceed $(c-1) \frac{k(k+1)}{2}+k$, where $c$ and $k$ are the classes of $G / N^{\prime}$ and $N$ respectively.

Later Steward showed in [6] that actually the class of $G$ in Theorem A is bounded by $(c-1)(k-1)+c k$. In the present paper however we are not concerned with explicit expressions for functions whose existence we are going to prove.

Hall's result proved to be an extremely useful criterion for a soluble group to be nilpotent. In particular the next theorem can be easily deduced from Theorem A.

THEOREM B. Let $\mathfrak{C}$ be a class of groups that is closed under taking subgroups and quotients. If all metabelian groups in $\mathfrak{C}$ are nilpotent, then so is any soluble group in $\mathfrak{C}$.

In [1], Endimioni and Traustason considered the question whether the above results remain true with "nilpotent" replaced by "torsion-by-nilpotent". They obtained the following analogue to Theorem B.

THEOREM C. Let $\mathfrak{C}$ be a class of groups that is closed under taking subgroups and quotients. If all metabelian groups in $\mathfrak{C}$ are torsion-by-nilpotent, then so is any soluble group in $\mathfrak{C}$.

The purpose of the present paper is to establish yet another analogue to Theorem B.

THEOREM 1.1. Let $\mathfrak{C}$ be a class of groups that is closed under taking subgroups and quotients. If any metabelian group in $\mathfrak{C}$ is an extension of a group of finite exponent by a nilpotent group, then so is any soluble group in $\mathfrak{C}$.

The above theorem is an immediate consequence of the following quantitative result. We use the term " $\{a, b, c, \ldots\}$-bounded" to mean "bounded from above by some function of $a, b, c, \ldots$.

Supported by CNPq-Brazil. 
THEOREM 1.2. Let $c, d, q$ be positive integers. Suppose $G$ is a soluble group with derived length $d$. Assume that for any $i$ the metabelian quotient $G^{(i)} / G^{(i+2)}$ is an extension of a group of finite exponent $q$ by a nilpotent group of class $c$. Then there exist $\{c, d, q\}$ bounded numbers $f$ and e such that $G$ is an extension of a group of finite exponent e by $a$ nilpotent group of class $f$.

In turn, Theorem 1.2 is deduced from the following analogue to Theorem A for soluble groups. We write $f(c, k)$ for the expression $(c-1) \frac{k(k+1)}{2}+k$.

THEOREM 1.3. Let $G$ be a group having a normal nilpotent of class $k$ subgroup $N$ such that $G / N^{\prime}$ is an extension of a group of finite exponent $q$ by a nilpotent group of class $c$. Assume that $\gamma_{c+1}(G)$ is soluble with derived length $d$. Then $\gamma_{f(k, c)+1}(G)$ has finite $\{c, d, k, q\}$-bounded exponent.

2. Preliminaries. As usual, if $M, N$ are subgroups of a group $G$, the subgroup $\langle[x, y] \mid x \in M, y \in N\rangle$ will be denoted by $[M, N]$. We use the left normed notation: thus if $N_{1}, N_{2}, \ldots, N_{i}$ are subgroups of $G$, then

$$
\left[N_{1}, N_{2}, \ldots, N_{i}\right]=\left[\ldots\left[\left[N_{1}, N_{2}\right], N_{3}\right], \ldots, N_{i}\right]
$$

The subgroup generated by all $n$th powers of elements of $M$ will be denoted by $M^{n}$. It follows that $M^{n r} \leq\left(M^{n}\right)^{r}$ for any $n, r$. For positive integers $d$ and $q$ we define the function $e(d, q)$ by the rule $e(d, q)=q^{d}$, if $q$ is odd and $e(d, q)=2^{d} q^{d}$, if $q$ is even.

LEMMA 2.1. Let $N$ be a soluble normal subgroup of a group $G$ and assume that $N$ has derived length $d$. Then for any $q$ we have $[N, G]^{e(d, q)} \leq\left[N^{q}, G\right]$.

Proof. Without any loss of generality we can assume that $\left[N^{q}, G\right]=1$. The claim is true if $N$ is abelian so we use induction on $d$. The induction hypothesis will be that $\left[N^{\prime}, G\right]^{e(d-1, q)}=1$. Passing to the quotient $G /\left[N^{\prime}, G\right]$ we can assume that $N^{\prime} \leq Z(G)$, in which case $N$ is nilpotent of class at most 2 and so for any $a \in N$ the map from $N$ to $N^{\prime}$ that takes an arbitrary element $x \in N$ to $[x, a]$ is a homomorphism whose kernel is $C_{N}(a)$. Because $\left[N^{q}, G\right]=1$, it follows that $N^{\prime}$ has exponent dividing $q$. Now the Hall-Petrescu formula [3, III.9.4] (see also the proof of Lemma VIII.1.1 (b) in [4]) gives us $[N, G]^{q} \leq\left[N^{q}, G\right] N^{\prime \frac{q}{2}}$ so $[N, G]$ has exponent dividing $q$ if $q$ is odd and $2 q$ otherwise. In either case the lemma follows.

Having fixed $d$ and $q$, for any $s \geq 1$ we set $e_{s}=e^{d^{s-1}}$, where $e=e(d, q)$.

COROLLARY 2.2. Under the hypotheses of Lemma 2.1 for any s we have

$$
[N, \underbrace{G, \ldots, G}_{s}]^{e_{s}} \leq[N^{q}, \underbrace{G, \ldots, G}_{s}] .
$$

Proof. This is straightforward by induction on $s$.

LEMMA 2.3. Let $N$ be a normal subgroup of a group $G$. Let $c, k$ be positive integers. Set $s=s(c, k)=k(c-1)+1$. Then we have

$$
[\underbrace{N, \ldots, N}_{k} \underbrace{G, \ldots, G}_{s}] \leq[[N, \underbrace{G, \ldots, G}_{c}], \underbrace{N, \ldots, N}_{k-1}] .
$$


Proof. We can assume that $[[N, \underbrace{G, \ldots, G}_{c}], \underbrace{N, \ldots, N}_{k-1}]=1$. In that case, of course, $[\underbrace{N, \ldots, N}_{m},[N, \underbrace{G, \ldots, G}_{c}], \underbrace{N, \ldots, N}_{k-1-m}]=1$ for any $m=0,1, \ldots, k-1$. Now write

$$
[\underbrace{N, \ldots, N}_{k} \underbrace{G, \ldots, G}_{s}] \leq \prod_{j_{1}+\cdots+j_{k}=s}\left[[N, \underbrace{G, \ldots, G}_{j_{1}}], \ldots,[N, \underbrace{G, \ldots, G}_{j_{k}}]\right] .
$$

This follows easily from equations (8) in [5, p. 117]. We notice that the number $s$ is big enough to ensure that at least one of the $j_{i}$ is bigger than or equal to $c$. Since $[\underbrace{N, \ldots, N}_{m},[N, \underbrace{G, \ldots, G}_{c}], \underbrace{N, \ldots, N}_{k-1-m}]=1$ for any $m=0,1, \ldots, k-1$, we derive that $[\underbrace{N, \ldots, N}_{k} \underbrace{G, \ldots, G}_{s}]=1$, as required.

3. Main Results. Now we are in a position to prove our main results. Since Theorem 1.1 is immediate from Theorem 1.2, it is sufficient to provide the proofs of Theorem 1.2 and Theorem 1.3. We let $f(c, k)$ stand for $(c-1) \frac{k(k+1)}{2}+k$ and $s=s(c, k)$ have the same meaning as in Lemma 2.3.

Proof of Theorem 1.3. We use induction on $k$. Set $H=\gamma_{f(k-1, c)+1}(G)$. The induction hypothesis is that the theorem holds if $G$ is replaced by $G / \gamma_{k}(N)$. In other words, we assume that there exists a $\{c, d, k, q\}$-bounded number $a$ such that $H^{a} \leq \gamma_{k}(N)$. Set $b=a^{d^{s(k, c)}}$. We will show that $\gamma_{f(k, c)+1}(G)$ has exponent dividing $b e_{k-1}$. We have

Notice that 2.2 shows that

$$
\left(\gamma_{f(k, c)+1}(G)\right)^{b e_{k-1}} \leq\left([H, \underbrace{G, \ldots, G}_{s}]^{b}\right)^{e_{k-1}} .
$$

$$
[H, \underbrace{G, \ldots, G}_{s}]^{b} \leq[H^{a}, \underbrace{G, \ldots, G}_{s}]
$$

so we write

$$
\left([H, \underbrace{G, \ldots, G}_{s}]^{b}\right)^{e_{k-1}} \leq[H^{a}, \underbrace{G, \ldots, G}_{s}]^{e_{k-1}} .
$$

Using that $H^{a} \leq \gamma_{k}(N)$, we obtain

Now Lemma 2.3 yields

$$
[H^{a}, \underbrace{G, \ldots, G}_{s}]^{e_{k-1}} \leq[\gamma_{k}(N), \underbrace{G, \ldots, G}_{s}]^{e_{k-1}} .
$$

$$
[\gamma_{k}(N), \underbrace{G, \ldots, G}_{s}]^{e_{k-1}} \leq[\gamma_{c+1}(G), \underbrace{N, \ldots, N}_{k-1}]^{e_{k-1}} .
$$

By 2.2 the latter expression is contained in $[\left(\gamma_{c+1}(G)\right)^{q}, \underbrace{N, \ldots, N}]$ while $\left(\gamma_{c+1}(G)\right)^{q} \leq$ $[N, N]$ by the hypothesis. Finally we write

$$
[\left(\gamma_{c+1}(G)\right)^{q}, \underbrace{N, \ldots, N}_{k-1}] \leq[N, N, \underbrace{N, \ldots, N}_{k-1}]=\gamma_{k+1}(N)=1 .
$$

Thus, we have shown that $\left(\gamma_{f(k, c)+1}(G)\right)^{b e_{k-1}}=1$, as required. 
Proof of Theorem 1.2. Since $G^{\prime}$ has derived length $d-1$, we can assume by induction on $d$ that there exist $\{c, d, q\}$-bounded numbers $k_{0}$ and $e_{0}$ such that $G^{\prime}$ is an extension of a group of finite exponent $e_{0}$ by a nilpotent group of class $k_{0}$. In particular, $\gamma_{k_{0}+1}\left(G^{\prime}\right)$ has exponent dividing $e_{0}$. Passing to the quotient-group $G / \gamma_{k_{0}+1}\left(G^{\prime}\right)$ we can assume without any loss of generality that $G^{\prime}$ is nilpotent of class at most $k_{0}$. By the hypothesis $\gamma_{c+1}\left(G / G^{\prime \prime}\right)$ has exponent dividing $q$. So, applying Theorem 1.3 with $N=G^{\prime}$, we obtain that $\gamma_{f\left(k_{0}, c\right)+1}(G)$ has finite $\{c, d, q\}$-bounded exponent.

\section{REFERENCES}

1. G. Endimioni and G. Traustason, On torsion-by-nilpotent groups, J. Algebra 241 (2001), 669-676.

2. P. Hall, Some sufficient conditions for a group to be nilpotent, Illinois J. Math. 2 (1958), 787-801.

3. B. Huppert, Endliche Gruppen I (Springer-Verlag, 1967).

4. B. Huppert and N. Blackburn, Finite groups II (Springer-Verlag, 1982).

5. D. J. S. Robinson, Finiteness conditions and generalized soluble groups, Part 1 (SpringerVerlag, 1972).

6. A. G. R. Steward, On the class of certain nilpotent groups, Proc. Roy. Soc. Ser A 292 (1966), 374-379. 